\title{
EXTENSIONS OF TORSION-COMPLETE GROUPS ${ }^{1}$
}

\author{
PAUL HILL AND CHARLES MEGIBBEN
}

Abstract. Problem 54 in L. Fuch's Infinite abelian groups. Vol. II, is solved negatively. An abelian p-group without elements of infinite height can be an extension of one torsion-complete group by another without itself being torsion complete.

As the abstract indicates, our main purpose is to resolve Problem 54 in [1], which will be referred to hereafter merely as Problem 54. Although we give a quick enough solution to the problem, the solution could be presented even more expediently. However, we shall enlarge the scope of the paper slightly and present some results that are not required for the solution of Problem 54 but are of independent interest.

Following L. Fuchs in [1], we say that an abelian p-group is separable if it has no elements of infinite height. Of particular interest to us will be the exact sequence

$$
H \rightarrow G \rightarrow Z\left(p^{\infty}\right),
$$

where $H$ is a torsion-complete $p$-group.

Proposition 1. If $H$ is torsion complete in the exact sequence (1), then $\operatorname{Pext}\left(Z\left(p^{\infty}\right), G\right)=0$. In particular, $G$ is torsion complete if it is reduced.

Proof. From (1), we obtain the exact sequence

$$
\operatorname{Hom}\left(Z\left(p^{\infty}\right), Z\left(p^{\infty}\right)\right) \rightarrow \operatorname{Ext}\left(Z\left(p^{\infty}\right), H\right) \rightarrow \operatorname{Ext}\left(Z\left(p^{\infty}\right), G\right) .
$$

Since $\operatorname{Hom}\left(Z\left(p^{\infty}\right), Z\left(p^{\infty}\right)\right)$ is isomorphic to the group of $p$-adic integers, it is compact in its $p$-adic topology. Moreover its image is compact in $\operatorname{Ext}\left(Z\left(p^{\infty}\right), H\right)$ since homomorphisms are always continuous with respect to the $p$-adic topologies. But $\operatorname{Pext}\left(Z\left(p^{\infty}\right), H\right)=0$ and therefore $\operatorname{Ext}\left(Z\left(p^{\infty}\right), H\right)$ is Hausdorff in its $p$-adic topology. Thus, the image of $\operatorname{Hom}\left(Z\left(p^{\infty}\right), Z\left(p^{\infty}\right)\right)$ is closed in $\operatorname{Ext}\left(Z\left(p^{\infty}\right), H\right)$, which means that the

Received by the editors June $18,1973$.

AMS (MOS) subject classifications (1970). Primary 20K10, 20K35; Secondary $20 \mathrm{~K} 40$.

Key words and phrases. Torsion-complete groups, extensions, abelian p-groups, Fuch's Problem 54.

${ }^{1}$ This research was supported by NSF grants GP-29025 and GP-3023X. 
corresponding quotient group is without elements of infinite height, that is, $\operatorname{Pext}\left(Z\left(p^{\infty}\right), G\right)=0$.

With $H$ torsion complete in (1), we see from Proposition 1 that $G$ is a direct sum of a divisible group and a torsion-complete $p$-group. Our next result tells us precisely when $G$ is reduced. For its statement we find it convenient to view $H$ as a subgroup of $G$.

Proposition 2. If $H$ is a reduced p-group, then the following conditions are equivalent:

(i) The exact sequence (1) represents an element of finite order in $\operatorname{Ext}\left(Z\left(p^{\infty}\right), H\right)$.

(ii) $G$ is not reduced.

(iii) $G=H+D$ where $D \cong Z\left(p^{\infty}\right)$.

Proof. Assuming (i), we see from Theorem 1 in [2] that there is an integer $n$ and a subgroup $C$ of $G$ such that

$$
G / H\left[p^{n}\right]=H / H\left[p^{n}\right] \oplus C / H\left[p^{n}\right] .
$$

But then $C / H\left[p^{n}\right] \cong Z\left(p^{\infty}\right)$, and since $H\left[p^{n}\right]$ is bounded it is easily seen that $C$ contains a nontrivial divisible subgroup.

Assuming (ii), we see that $G$ contains a subgroup $D \cong Z\left(p^{\infty}\right)$. As $H$ is reduced, $D$ must map onto $Z\left(p^{\infty}\right)$ under the homomorphism $G \rightarrow Z\left(p^{\infty}\right)$. Since $H$ is the kernel of this map, $G=H+D$.

Finally, assume (iii). Then $G / X=H / X \oplus D / X$ where $X=H \cap D$. There is then an integer $n$ such that $X$ is a cyclic group of order $p^{n}$. It follows that $H\left[p^{n}\right]=X \oplus L$ for some subgroup $L$ of $H$. If we set $C=D+L$, it is readily seen that we have the direct decomposition (2) again. Another application of Theorem 1 in [2] yields the conclusion that (1) represents an element of finite order in $\operatorname{Ext}\left(Z\left(p^{\infty}\right), H\right)$.

COROLlary. If $H$ is a torsion-complete p-group, then $G$ is torsion complete if and only if (1) represents an element of infinite order in $\operatorname{Ext}\left(Z\left(p^{\infty}\right), H\right)$.

As $\operatorname{Ext}\left(Z\left(p^{\infty}\right), H\right)$ is torsion only when $H$ is bounded, we see that there is an abundance of reduced groups $G$ fitting into exact sequences of the form (1). The relevance of our preceding results to Problem 54 is made clear by our next proposition.

Proposition 3. The existence of the exact sequence (1) with $H$ torsion complete and $G$ separable is equivalent to the existence of an exact sequence

$$
A: \rightarrow C \rightarrow B,
$$


where $A$ and $B$ are torsion-complete p-groups and $C$ is separable but not torsion complete.

Proof. Assume the existence of (3), and let $\bar{C}$ denote the torsion completion of $C$. Then $\bar{C} / C \cong \sum_{r} Z\left(p^{\infty}\right)$ with $r \geqq 1$ since $C \neq \bar{C}$. Let $C^{*}$ be a subgroup of $\bar{C}$ containing $C$ such that $C^{*} / C=Z\left(p^{\infty}\right)$. Due to the purity of $C$ in $C^{*}$ and the fact that $C \mid A \cong B$ is torsion complete, we have the decomposition

$$
C^{*}|A=C| A \oplus G \mid A,
$$

where $G \subseteq C^{*} \subseteq \bar{C}$ is a separable $p$-group. Since $G \mid A \cong C^{*} / C=Z\left(p^{\infty}\right)$, we have obtained the exact sequence (1) with $H=A$ torsion complete and $G$ separable. Proposition 1 implies that $G$ is actually torsion complete.

Conversely, suppose that we start with the exact sequence (1) with $H$ torsion complete and $G$ separable (and therefore also torsion complete). Choose $K$ to be a pure subgroup between an unbounded basic $p$-group $B$ and its torsion completion $\bar{B}$ so that $\bar{B} \mid K=Z\left(p^{\infty}\right)$. By the choice of $K$, it is not torsion complete. Let $S$ be the subdirect sum of $G$ and $\bar{B}$ corresponding to the isomorphisms $G / H \cong Z\left(p^{\infty}\right) \cong \bar{B} / K$. Then $S \subseteq G \oplus \bar{B}$ is obviously separable, and we have the exact sequence

$$
H: \rightarrow S \rightarrow \bar{B} .
$$

It remains only to show that $S$ is not torsion complete, but this follows immediately from the purity of $K$ in $S$ and the exact sequence

$$
K \longmapsto S \rightarrow G .
$$

Indeed a closed, pure subgroup of a torsion-complete group is itself torsion complete and, of course, $K$ is not torsion complete.

Combining our foregoing results, we have

THEOREM. A separable p-group $G$ does not have to be torsion complete in order to be the extension of a torsion-complete group $H$ by a torsioncomplete group $K$. However, if not, the torsion completion $G$ of such a group $G$ must contain a subgroup $M$ that contains $H$ as a proper dense subgroup.

The final assertion in our theorem follows from (4) in the proof of Proposition 3.

COROLlaRY Suppose that $H \subseteq G \subseteq L$ where $L$ is torsion complete and $L / H$ is reduced. Then $G$ is torsion complete if $G$ is pure in $L$ and $G / H$ is torsion complete. 
Proof. By Corollary 68.7 in [1], $H$ is torsion complete. Thus $G$ is the extension of the torsion-complete group $H$ by the torsion-complete group $G / H$. The torsion incompleteness of $G$ would imply, according to the Theorem, that $\bar{G} / H$ is not reduced. However, $\bar{G} / H$ is reduced since $L / H$ is reduced and $\bar{G} \subseteq L$.

REMARKS. Most of the foregoing results are not essential to a solution of Problem 54. Indeed all that is actually required is one half of Proposition 3 and some exact sequence (1) with $H$ torsion complete and $G$ separable. The construction of such an extension can be accomplished as follows. Take $B=\bigoplus_{i=1}^{\infty}\left\langle b_{i}\right\rangle$ where $b_{i}$ has order $p^{2 i}$ and set $a_{i}=b_{i}-p b_{i+1}$. Let $H$ be the torsion completion of $A=\bigoplus_{i=1}^{\infty}\left\langle a_{i}\right\rangle$. Then $H$ can be viewed in a natural manner as a subgroup of $\bar{B}$, the torsion completion of $B$. Note that $b_{i} \notin H$. Indeed if $b_{i}=\sum_{i=1}^{\infty} t_{i} a_{i}$, then the sequence $\left\{\sum_{i=1}^{n} t_{i} a_{i}\right\}$ is unbounded. Take $G=B+H$. $G$ is separable since it is subgroup of $\bar{B}$ and $G / H \cong Z\left(p^{\infty}\right)$ since $B / A \cong Z\left(p^{\infty}\right)$.

Finally we remark without proof that a restricted version of Problem 54 has an affirmative answer. Indeed if $H \leftrightarrow G \rightarrow K$ represents an element of finite order in $\operatorname{Ext}(K, H)$ with $H$ and $K$ torsion complete and $G$ separable, then $G$ is necessarily torsion complete.

\section{REFERENCES}

1. L. Fuchs, Infinite abelian groups. Vol. II, Academic Press, New York, 1973.

2. C. Walker, Properties of Ext and quasi-splitting of abelian groups, Acta Math. Acad. Sci. Hungar. 15 (1964), 157-160. MR 29 \#4798.

Department of Mathematics, Florida State University, Tallahassee, Florida 32306

Department of Mathematics, Vanderbilt University, Nashville, Tennessee 37235 